\title{
Peculiarities of Radical Homo- and Copolymerization of Acrylic, Methacrylic and Itaconic Acids in Complexing Solutions
}

\author{
Gerardas BAJORAS and Ričardas MAKUŠKA \\ Department of Polymer Chemistry, Vilnius University, \\ Vilnius 232600, USSR
}

(Received January 16, 1986)

\begin{abstract}
Using the method of IR spectroscopy a study was made of complexation in the systems of acrylic, methacrylic and itaconic acids-dimethyl sulphoxide-water. Quantitative assessment was done on the number of acid molecules entering the $\mathrm{H}$-bonds with DMSO using different amounts of water in the systems. Calculations were performed on the parameters of electron structure of itaconic acid, establishing higher acidity of the $\alpha$-carboxylic group. Reactivity of methacrylic acid in different complexes was assessed according to the redistribution of electron density in monomer molecules and in acid radicals during complexation. Copolymerization of acrylic, methacrylic and itaconic acids with acrylo- and methacrylonitriles in solutions of dimethyl sulphoxide-water was investigated as well. Peculiarities and differences of copolymerization of itaconic acid and monocarboxylic acids in binary solvents are thoroughly discussed here.

KEY WORDS Radical Polymerization / Copolymerization / Acrylic Acid / Methacrylic Acid / Itaconic Acid / Acrylonitrile / Methacrylonitrile / Complexing Solutions / IR Spectra / Electron Density /
\end{abstract}

It is well known that electron-donor solvents diminish reactivity of unsaturated carboxylic acids during homo- and copolymerization. ${ }^{1-5}$ Almost all researchers consider the formation of H-complexes between monomer molecules or growing macroradicals and the components of the reaction medium to be the main cause of this phenomenon. Any possibility to assess each case of complexation in reaction systems is of great importance for controlling the parameters of polymerization. However, direct investigations of complexation betwen monomers and solvents are very few ${ }^{6-10}$ as yet and it is difficult to make judgement on the role of H-complexes, particularly in complex reaction systems using mixtures of aprotonic and protonic solvents. An alternative mechanism involving the activity of acids during complexation has also been studied insufficiently.
In this paper an attempt has been made to investigate and describe the complexation of acrylic acid (AA), methacrylic acid (MAA), and itaconic acid (IA) in a mixed dimethyl sulphoxide (DMSO)-water solvent trying to establish the most reliable causes of propagation reactivity and to evaluate the influence of complexation on homo- and copolymerization of these monomers.

\section{EXPERIMENTAL}

AA and MAA were purified by crystallization followed by double distillation under reduced pressure. The following fractions were collected: AA (bp $314-315^{\circ} \mathrm{C} / 1.6 \mathrm{kPa}, n_{\mathrm{D}}^{20}$ $1.4224)$, MAA (bp $328-239^{\circ} \mathrm{C} / 0.93 \mathrm{kPa}, n_{\mathrm{D}}^{20}$ 1.4316). IA was twice recrystallized from ethanol (bp 436-438.K), the purity of IA being not less than $98 \%$, Acrylonitrile (AN) 
and methacrylonitrile (MAN) were washed from stabilizer successively with a $10 \%$ aqueous solution of $\mathrm{NaOH}$ and distilled water till neutral reaction was obtained, followed by drying over anhydrous $\mathrm{CaCl}_{2}$ and by fractional distillation. The following fractions were collected: AN (bp $349.6-350.2^{\circ} \mathrm{C}, n_{\mathrm{D}}^{20}$ 1.3935), MAN (bp $363-364^{\circ} \mathrm{C}, n_{\mathrm{D}}^{20} 1.3975$ ).

In this work we have used DMSO dried over anhydrous $\mathrm{CaCl}_{2}$ and distilled under vacuum (bp $335-336^{\circ} \mathrm{C} / 0.5 \mathrm{kPa}, n_{\mathrm{D}}^{20} 1.4783$ ), distilled water, and azobisisobutyronitrile (AIBN) initiator $\left(\mathrm{mp} 374-375^{\circ} \mathrm{C}\right)$.

Complexation in reaction systems was assessed spectrometrically, the IR spectra being recorded by a UR-20 spectrophotometer. The parameters of electron structure of $\mathrm{AA}$ and MAA molecules and radicals in various complexes were calculated by the method of $\mathrm{CNDO} / 2$, and those of IA molecules by MINDO/3 method. ${ }^{11}$ The length of bonds in monomers and in their radicals as well as the angles between the bonds are the standard values given in the literature. ${ }^{12,13}$ Optimal space structure of MAA radicals in the complexes was calculated according to their minimal energy obtained by changing the space orientation of $\mathrm{COOH}$ and $\mathrm{CH}_{3}$ groups. Calculations were made for growing radicals, i.e., upon addition of a second monomeric unit to the hypothetical primary radical. Copolymerization of unsaturated carboxylic acids with AN and MAN in DMSO-water solution was carried out in glass-sealed ampoules in nitrogen or argon atmosphere at $343 \pm 0.1^{\circ} \mathrm{C}$, the total monomer concentration in $\mathrm{AA}$ and MAA copolymerization being $3.75 \mathrm{~mol} \mathrm{dm}^{-3}$ and in case of IA copolymerization $3.3 \mathrm{~mol}$ $\mathrm{dm}^{-3}$. The concentration of the initiator (AIBN) was $0.016 \mathrm{~mol} \mathrm{dm}^{-3}$. The resulting copolymers were precipitated from the reaction mixture using acidified water (1 N $\mathrm{HCl}$ ), and its mixture with diethyl ether for monomer compositions enriched on acids, followed by wash-outs with distilled water and drying in vacuum to constant weight. The conversion of monomers was established gravimetrically, the composition of copolymers being determined according to the nitrogen content by the Kjeldahl method and according to the carboxyl groups, while the reactivity ratios of comonomers were calculated by the analytic method. ${ }^{14}$ The rate of polymerization of AA in binary solvent was measured dilatometrically, the contraction fractor of monomer (at $343^{\circ} \mathrm{C}$ ) being 0.24 .

\section{DISCUSSION AND RESULTS}

In order to determine the role of complex formation in polymerization of unsaturated carboxylic acids in the mixture of DMSOwater it is necessary to consider their interrelations as reflected in the IR spectra starting from simple binary systems.

In the spectrum of diluted AA solution in nonpolar $\mathrm{CCl}_{4}$, the greatest intensity is observed in the complex band $v_{\mathrm{OH}}$, involving the region at $2400-3400 \mathrm{~cm}^{-1}$ (Figure 1) and the band $v_{\mathrm{C}=\mathrm{O}}$ at $1704 \mathrm{~cm}^{-1}$ (Figure 2), the band corresponding to the dimer of the acid. Even at<smiles>[R]C(=O)O[Hg]OC([R])=O</smiles>

concentrations of $0.2-0.5 \mathrm{~mol} \mathrm{dm} \mathrm{dm}^{-3}$ the number of free molecules of the acid is small

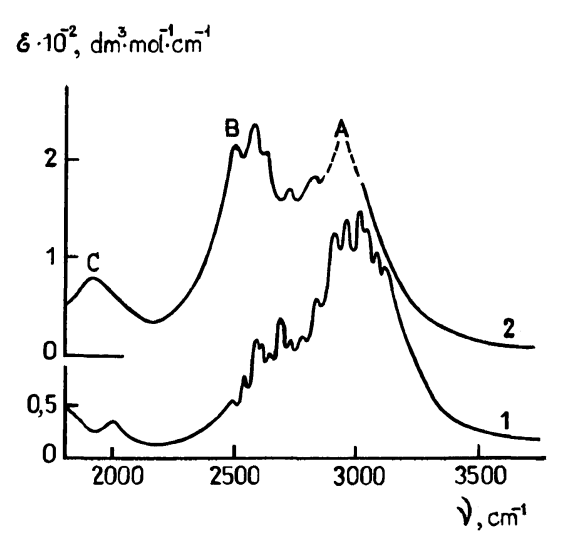

Figure 1. IR spectra ( $v_{\mathrm{OH}}$ region) of $\mathrm{AA}$ in $\mathrm{CCl}_{4}$ (1) and DMSO (2). $[\mathrm{AA}]=0.46 \mathrm{~mol} \mathrm{dm}^{-3}$. 


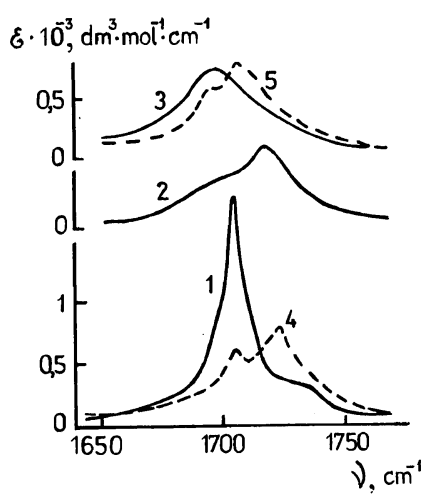

Figure 2. IR spectra ( $\xi_{\mathrm{C}=\mathrm{O}}$ region) of $\mathrm{AA}$ in $\mathrm{CCl}_{4}$ (1), DMSO (2), $\mathrm{D}_{2} \mathrm{O}(3), 97.8 \% \mathrm{CCl}_{4}+2.2 \%$ DMSO (4), and $24 \% \mathrm{D}_{2} \mathrm{O}+76 \%$ DMSO (5). [AA] $=0.46 \mathrm{~mol} \mathrm{dm}^{-3}$.

$(<1 \%)$ and their corresponding bands $v_{\mathrm{c}=\mathrm{c}}$ $1750 \mathrm{~cm}^{-1}$ and $v_{\mathrm{OH}} 3530 \mathrm{~cm}^{-1}$ in the spectrum are not observed.

In the spectrum of AA in DMSO medium a complex band consisting of three components $\mathrm{A}, \mathrm{B}, \mathrm{C}$, which are characteristic of a very strong $\mathrm{H}$-bond ${ }^{15}$ can be observed in the absorption region of $v_{\mathrm{OH}}$ (Figure 1). In the $v_{\mathrm{c}=\mathrm{O}}$ range there is a double band with its maximum at $1716 \mathrm{~cm}^{-1}$, corresponding to the complex

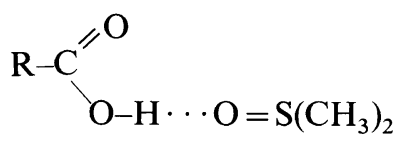

The band is slightly shifted to high frequencies with respect to the $v_{\mathrm{c}=\mathrm{o}}$ band of dimers, so that the carbonyl group of the acid does not participate in the $\mathrm{H}$-bond. The cause of splitting of the $v_{\mathrm{c}=\mathrm{o}}$ band of the complex is not clear as yet, though such phenomenon has been observed before ${ }^{16,17}$ while investigating the spectra of complexes of acids with strong proton acceptors (pyridine, amine).

Investigating the IR spectra of AA in water we used $\mathrm{D}_{2} \mathrm{O}$, because $\mathrm{H}_{2} \mathrm{O}$ is a violent absorbent in the $v_{\mathrm{c}=\mathrm{O}}$ range of acid. Aqueous solution is characterized by increased intensity, a half-width of the $v_{\mathrm{c}=\mathrm{O}}$ band, and its shift to the lower frequencies in respect to $v_{\mathrm{c}}=\mathrm{O}$ dimers. This testifies to the fact that the in- teraction of AA with water occurs instantaneously via the two functional groups of acid with formation of $\mathrm{H}$-complexes<smiles>[R]C(=O)O[Hg]O</smiles>

The spectrum of the solution of AA in mixture of $\mathrm{CCl}_{4}$ and DMSO is similar to that of the solution of AA in DMSO (Figure 2). Certain differences in the location of the maximum of $v_{\mathrm{c}=\mathrm{O}}$ band of the H-complex of AA $\cdots$ DMSO in $\mathrm{CCl}_{4}$ and directly in DMSO are conditioned by a different polarity of these solvents ( $\mu=0$ and 3.9, respectively). A greater part of acid molecules interacts with DMSO, but not with each other. This is to be expected because the enthalpy $|\Delta H|$ of the formation of $\mathrm{H}$-bonds in dimer is equal to $21-23 \mathrm{~kJ}$ $\mathrm{mol}^{-1}$, while that of the complex of aliphatic acids with DMSO is $40 \mathrm{~kJ} \mathrm{~mol}^{-1} \cdot{ }^{18}$ When one of the dimers is broken and two complexes of acid-DMSO are formed, extra $34-38 \mathrm{~kJ}$ $\mathrm{mol}^{-1}$ are discharged.

In the spectrum of AA solution in a mixture of DMSO and $\mathrm{D}_{2} \mathrm{O}$ one can see $v_{\mathrm{c}=\mathrm{o}}$ band, its shape and location testifying the interaction of acid with water and DMSO. Apart from the complex

$$
\begin{aligned}
& \mathrm{R}-\mathrm{C}^{\prime \prime} \mathrm{O} \cdot \mathrm{H}-\mathrm{O}^{-\mathrm{H}} \\
& \mathrm{O}-\mathrm{H} \cdot \mathrm{O}=\mathrm{S}\left(\mathrm{CH}_{3}\right)_{2},
\end{aligned}
$$

complex III and some other more complex aggregates can find realization here.

The basic scheme of complexation of MAA is essentially similar to that of AA and it is pointless to describe it in detail. IA does not dissolve in $\mathrm{CCl}_{4}$. In the $v_{\mathrm{c}=\mathrm{O}}$ range of the spectrum of the solution of IA in acetonitrile $\left(\mathrm{CD}_{3} \mathrm{C} \equiv \mathrm{N}, \mathrm{ACN}\right)$ one can see a wide absorption band in the form of twin peaks with components at 1728 and $1748 \mathrm{~cm}^{-1}$ (Figure 3). 


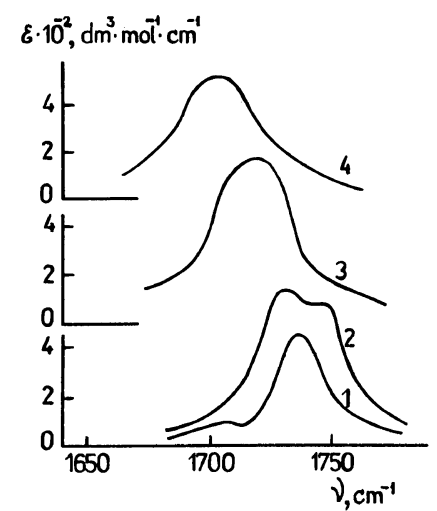

Figure 3. IR-spectra $\left(v_{\mathrm{C}=\mathrm{O}}\right.$ region) of $\mathrm{AA}(1)$ and IA $(2-4)$ in $A C N(1,2)$, DMSO (3), and $\mathrm{D}_{2} \mathrm{O}$ (4). [AA] $=$ $0.36 \mathrm{~mol} \mathrm{dm}^{-3},[\mathrm{IA}]=0.18 \mathrm{~mol} \mathrm{dm}^{-3}$.

The total half-width $\Delta v_{1 / 2}$ of this complex band is abnormally large making $45 \mathrm{~cm}^{-1}$. It markedly exceeds the half-width of the $v_{\mathrm{c}}=\mathrm{o}$ band of aliphatic saturated and unsaturated acids(propionic, acrylic, methacrylic) and dibasic acids with equally valuable carboxylic groups (malonic, succinic, adipinic) for which $\Delta v_{1 / 2}$ is $17-30 \mathrm{~cm}^{-1}$. In the spectrum of IA in DMSO the splitting of $v_{\mathrm{c}}=\mathrm{o}$ band is not observed, though its half-width is $20-30 \%$ larger than the value of $\Delta v_{1 / 2}$ of $v_{\mathrm{c}=0}$ band obtained from other acids mentioned above and this probably depends on the complex structure of $v_{\mathrm{c}=\mathrm{o}}$ band.

It is known ${ }^{19}$ that the constants of dissociation of carboxylic groups of IA differ by two orders of value $\left(K_{1}=1.5 \times 10^{-4}, K_{2}=2.2 \times\right.$ $\left.10^{-6}\right)$. It has been accepted ${ }^{20}$ that $\beta$-carboxylic group IA possesses greater acidity and is more readily dissociated

$$
\mathrm{CH}_{2}=\mathrm{C}^{\prime}{ }^{\alpha} \mathrm{COOH}
$$

However, a simple comparison of the acidic properties of the corresponding saturated and unsaturated carboxylic acids gives rise to some doubts concerning the statement. Our analysis of the electron structure of IA (see Scheme) has revealed that the $\alpha-\mathrm{COOH}$ group possesses greater polarity, and naturally, it has greater ability for proton splitting. The acidity of a less polar $\beta$-COOH group is lower. In the range of proton donors containing hydroxyl groups, including carboxylic acids, the ability of molecules to split the proton (acidity) and to form hydrogen bonds (proton donor ability) undergoes symbatic changes. ${ }^{21}$ It is quite natural to expect the proton donor ability of $\alpha-\mathrm{COOH}$ group of IA to be higher than that of $\beta$ - $\mathrm{COOH}$ group, i.e., in IA complexes with acceptors of protons $\mathrm{B}$ (ACN or DMSO molecules) the $v_{\mathrm{c}=\mathrm{O}}$ absorption bands should become wider or even split.

There are no spectral features showing the presence of IA dimers in ACN or DMSO solutions, while the spectrum of AA in ACN solution (Figure 3 ) shows a slight band at $1707 \mathrm{~cm}^{-1}$ which is attributed to acid dimers. On analyzing the location and form of the $v_{\mathrm{c}}=\mathrm{O}$ absorption band of IA in these solutions it is possible to assume the existence of two types of complexes:<smiles>C=C(CC(=O)O)C(=O)P</smiles>

(VII)

higher acidity forms a stronger bond with the electron donor molecule B. The VII complex of $1: 1$ composition has the structure with an 


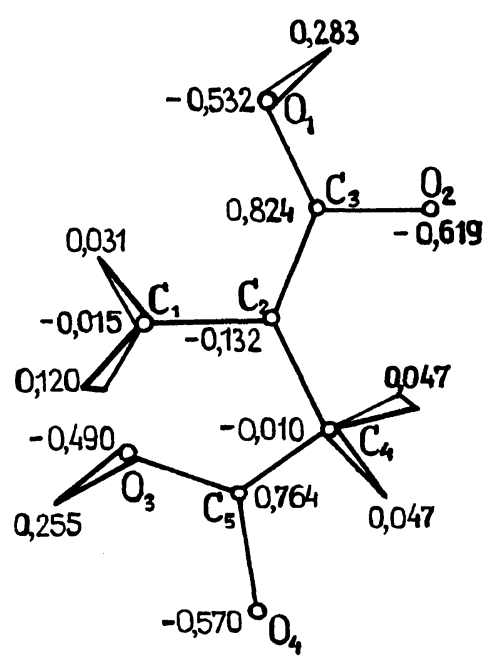

Scheme 1. Space structures and atomic charge distribution of IA molecule.

intramolecular $\mathrm{H}$-bond. The possibility of realization of such intermolecular $\mathrm{H}$-bonds has been reported earlier. ${ }^{22}$ It depends on the fact that in highly polar solvents energetically more advantageous is the trans-conformation of $\mathrm{C}=\mathrm{O}$ and $\mathrm{OH}$ groups into $\alpha-\mathrm{COOH}$, whose dipole moment is higher than that of cisisomer. ${ }^{23}$ Both types of complexes (VI and VII) are in equilibrium with each other, the equilibrium being shifted to the side of complex VI in the case of IA solution in DMSO, whose molecules are more powerful proton acceptors than ACN. One can expect that the low frequency component of $v_{\mathrm{c}=\mathrm{o}}$ band of IA in ACN depends on the absorption of carbonyl present in the cycle of intramolecular $\mathrm{H}$-bond, because in this case the very $\mathrm{C}=\mathrm{O}$ group directly participates in the interaction. Inequality of $\mathrm{H}$-bonds in the VI complex in $\alpha$ $\mathrm{COOH}$ and $\beta-\mathrm{COOH}$ groups is probably reflected only on the widening of the band.

The spectrum of IA in the $v_{\mathrm{c}}=\mathrm{o}$ range in water is similar to the spectra of AA and MAA (Figures 2, 3). There is no doubt that IA interacts with water molecules in all functional groups.<smiles>C=C1CC(=O)O[C@H](O)O[C@@H]1O</smiles>

(VIII)

Analytic investigations of complexation of acids appear to be feasible according to the intensity of " $\mathrm{C}$ " peak of $v_{\mathrm{OH}}$ band of AA or MAA $\left(1900 \mathrm{~cm}^{-1}\right)$ forming strong $\mathrm{H}$-bond complexes with DMSO, and also according to the $v_{\mathrm{c}=\mathrm{o}}$ band shift towards the low frequency upon increase of water in the composition of mixed solution.

Figure 4 shows the dependences of the apparent (measurable) absorption coefficients $\varepsilon$ of the "C" peak of $v_{\mathrm{OH}}$ band $\left(1900 \mathrm{~cm}^{-1}\right)$ on the concentration of AA and MAA in DMSO-water solutions. It is evident that with increase in acid concentration, $\varepsilon$ decreases. This means that not all acid molecules form complexes with DMSO, part of the molecules remain bound with each other, forming dimers.

Extrapolating curves 1 and 3 to zero concentration of AA and MAA, it is possible to

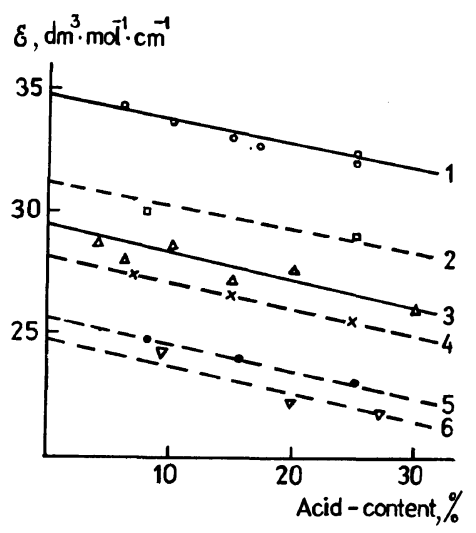

Figure 4. Dependence of apparent absorption coefficient $\varepsilon$ of the C peaks $\left(1900 \mathrm{~cm}^{-1}\right)$ of $v_{\mathrm{OH}}$ bands of the complexes AA-DMSO $(1,2,4,5)$ and MAA-DMSO $(3,6)$ on the concentration of acids. DMSO : $\mathrm{H}_{2} \mathrm{O}$, mass $\%: 1,3,100: 0 ; 2,6,90: 10 ; 4,80: 20 ; 5,75: 25$. 
obtain the true value of $\varepsilon=\varepsilon_{\mathrm{o}}$ of the band. Thus, for these curves the ratio of $\varepsilon / \varepsilon_{\mathrm{o}}$ at any acid concentration up to $25-30 \%$ is equal to the part of molecules bound with DMSO, while $\left(1-\varepsilon / \varepsilon_{\mathrm{o}}\right)$ refers to the molecules bound in dimers. Thus, at concentration of $25 \%$, more than $90 \%$ of acid molecules form $\mathrm{H}$-bonds with DMSO and only $7-9 \%$ remain bound in dimers. This agrees well with the cryoscopically assessed amount of dimerized AA in DMSO solutions. ${ }^{24}$

The decrease of $\varepsilon$ on addition of a certain amount of water into solution means that part of $\mathrm{H}$-bonds $\mathrm{R}$ - $\mathrm{COOH} \cdots \mathrm{O}=\mathrm{S}\left(\mathrm{CH}_{3}\right)_{2}$ is replaced by $\mathrm{R}-\mathrm{COOH} \cdots \mathrm{OH}_{2}$ bonds, forming not only complex IV, but also complex III. Thus, for the system of $25 \%$ of AA in mixture of $25 \%$ of $\mathrm{H}_{2} \mathrm{O}$ and $75 \%$ of DMSO $\varepsilon / \varepsilon_{0}=$ $0.67 \pm 0.02$; consequently, about $65-69 \%$ of acid molecules are bound with DMSO through $\mathrm{OH}$ groups, and only $31-35 \%$ of molecules

$$
\mathrm{R}-\mathrm{C}=\mathrm{O}-\mathrm{H} \cdots \mathrm{O}=\mathrm{S}\left(\mathrm{CH}_{3}\right)_{2}
$$

For this equilibrium $K_{2} \approx 12$. Thus, $K_{2}>K_{1}$, almost by one order, i.e., the second process is more feasible.

For $25 \%$ solution of AA in mixture of $75 \%$ DMSO- $25 \% \quad \mathrm{H}_{2} \mathrm{O}$ the equilibrium between complexes IV and III can be defined using constant $K_{3} \approx 0.4$. However, this system may contain complexes of other types, therefore the given equilibrium is not unique and our attempts at describing it are speculative.

For the purpose of investigation of complexation of acids in mixture of DMSO- $\mathrm{H}_{2} \mathrm{O}$ with excess of water, we have studied the influence of the composition of solvent on the frequency in the $\mathrm{C}=\mathrm{O}$ range of $\mathrm{AA}, \mathrm{MAA}$, and IA. The frequencies of $v_{\mathrm{c}=\mathrm{o}}$ bands in the spectrum of acid solutions in DMSO have greatest values $\left(1716 \mathrm{~cm}^{-1}\right.$ for AA, $1713 \mathrm{~cm}^{-1}$ for IA and $1704 \mathrm{~cm}^{-1}$ for MAA). On addition are bound with $\mathrm{H}_{2} \mathrm{O}$. In the corresponding MAA solution about $60-66 \%$ of acid molecules are bound with DMSO through $\mathrm{OH}$ groups.

From the spectral data using the results of Figure 4, it is possible to determine the equilibrium constants of the formation processes of different complexes in solution. Thus, for equilibrium between complexes I and II

$$
K_{1}=\frac{C_{\mathrm{k}}^{2}}{C_{\mathrm{d}} \cdot C_{\text {DMSO }}^{2}}
$$

where $C_{\mathrm{k}}, C_{\mathrm{d}}$, and $C_{\text {DMso }}$ are the concentrations of H-complexes of acid-DMSO, cyclic dimers of acid and free DMSO molecules, respectively. For $25 \%$ solution of $\mathrm{AA}$ in DMSO, which has about $8 \%$ acid molecules in dimers, $K_{1} \approx 1.6 \mathrm{dm}^{3} \mathrm{~mol}^{-1}$.

In this system there may be another process associated with the existence of open acid dimers, as follows<smiles>[R]C(=O)O[Hg]O[Si]</smiles>

of water $\left(\mathrm{D}_{2} \mathrm{O}\right)$, the band $v_{\mathrm{c}=\mathrm{O}}$ shifts to lower frequencies (Figure 5) due to the formation of

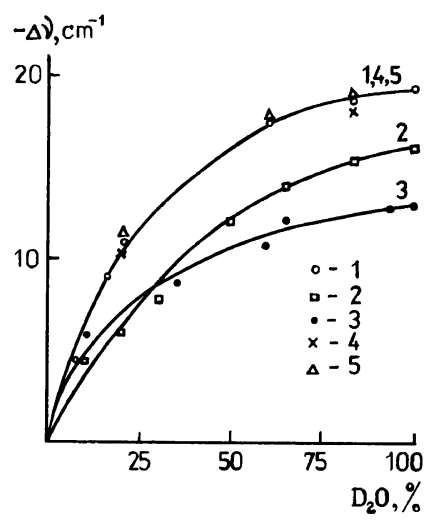

Figure 5. Dependence of the shifts to lower frequencies $\Delta v$ of $v_{\mathrm{C}=\mathrm{O}}$ bands of acids due to $\mathrm{D}_{2} \mathrm{O}$ content in the mixture DMSO- $\mathrm{D}_{2} \mathrm{O} .1,2 \% \mathrm{AA}$ at $298 \mathrm{~K} ; 2$, $2 \%$ MAA at $298 \mathrm{~K} ; 3,2 \%$ IA at $298 \mathrm{~K} ; 4,2 \%$ AA at $343 \mathrm{~K} ; 5,25 \%$ AA at $298 \mathrm{~K}$. 
complexes IV, III, VIII and more complex associates. There is no doubt that the value of low frequency shift of $v_{\mathrm{c}=\mathrm{O}}$ band is the measure of the influence of $\mathrm{H}$-bonds on the acid molecule.

It is necessary to point out that the solution temperature has little effect on the complexation of acids (Figure 5). Thus, shifts of $v_{\mathrm{c}}=\mathrm{o}$ band of AA, which are observed in DMSO$\mathrm{H}_{2} \mathrm{O}$ solutions at $298 \mathrm{~K}$ and $343 \mathrm{~K}$, are much alike in character. An increase in monomer concentration in solution from $2 \%$ to $25 \%$ has also little effect. Therefore, under conditions of polymerization in DMSO- $\mathrm{H}_{2} \mathrm{O}$ solutions, the complexation of acids should slightly differ from that one described above.

As discussed earlier, reactivity of unsaturated carboxylic acids during polymerization in DMSO (or in some other strong electrondonor solvents) decreases. However, addition of a small amount of water to acid solution in DMSO markedly increases the total rate of polymerization (Figure 6). It depends mainly on the fact that the propagation rate constant $k_{\mathrm{p}}$ upon addition of water into reaction mix- ture increases more readily than the constant of termination rate $k_{\mathrm{t}}$. Thus, on addition of $20 \%$ of $\mathrm{H}_{2} \mathrm{O}$ to AA solution $\left(1.79 \mathrm{~mol} \mathrm{dm}^{-3}\right)$ in DMSO, the $k_{\mathrm{p}}$ increases by six times, and $k_{\mathrm{t}}$ only by 1.5 times. $^{25}$ An increase in the polymerization reactivity of carboxylic acids in DMSO- $\mathrm{H}_{2} \mathrm{O}$ solutions is evidently associated with its competitive complexation. On addition of water to the reaction mixture, the number of acid molecules complexed with

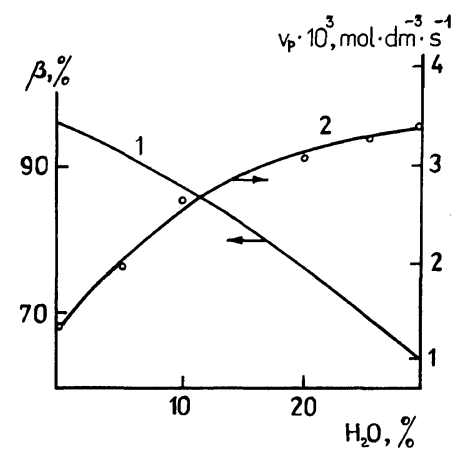

Figure 6. Dependence of the fraction $\beta$ of molecules of AA complexated with DMSO (1) and the initial rate of polymerization of AA on the composition of solvent DMSO- $\mathrm{H}_{2} \mathrm{O}$. $[\mathrm{AA}]=1.79 \mathrm{~mol} \mathrm{dm}^{-3}$.

Table I. Quantum-chemical characteristics of complexated acid molecules

\begin{tabular}{|c|c|c|c|c|}
\hline \multirow{2}{*}{ Complex } & \multicolumn{3}{|c|}{ Charge transfer between components of $\mathrm{H}$-complex } & \multirow{2}{*}{$\begin{array}{c}\text { Wiberg index of } \\
\mathrm{C}=\mathrm{C}, W\end{array}$} \\
\hline & Acid & $\mathrm{H}_{2} \mathrm{O}$ & $\mathrm{H}_{2} \mathrm{O}$ or DMSO & \\
\hline II & -0.117 & - & 0.117 & 1.365 \\
\hline IV & -0.052 & -0.072 & 0.124 & 1.354 \\
\hline III & -0.015 & -0.070 & 0.086 & 1.343 \\
\hline MAA & - & - & - & 1.339 \\
\hline AA & - & - & - & 1.366 \\
\hline
\end{tabular}

Table II. Quantum-chemical characteristics of complexated acid radicals

\begin{tabular}{lcccc}
\hline & \multicolumn{2}{c}{ Charge transfer between components of H-complex } & $\begin{array}{c}\text { Spin density of } \\
\text { a single electron, } \\
\text { Complex }\end{array}$ \\
\cline { 2 - 4 } & Acid & $\mathrm{H}_{2} \mathrm{O}$ & $\mathrm{H}_{2} \mathrm{O}$ or DMSO & $S$ \\
\hline II & -0.036 & - & 0.036 & 0.506 \\
IV & -0.023 & -0.046 & 0.069 & 0.474 \\
III & -0.045 & -0.048 & 0.093 & 0.473 \\
MAA & - & - & - & 0.500 \\
AA & - & - & - & 0.560 \\
\hline
\end{tabular}


DMSO decreases (Figure 6), and this may be a decisive factor in altering its activity. Analysis of the possible causes of changes in reactivity of acids during their competitive complexation gives ground to suggest that redistribution of electron density in complexes of molecules and radicals in responsible for these changes.

Quantum-chemical calculations have shown that the greatest transference of electron density to the molecule of MAA occurs during. its complexation with DMSO (Table I). In complexes IV and III the negative charge of acid molecules is markedly lower. However, during complexation of MAA radicals the transfer of the charge on to the radical in complexes II-IV is approximately similar (Table II). These findings give reason to suggest that the formation of $\mathrm{H}$-complexes in reaction medium of DMSO- $\mathrm{H}_{2} \mathrm{O}$ markedly affects the activity of acid molecules more than that of its radicals.

The Wiberg index characterizes the firmness of bonds ${ }^{26}$ and to some degree it reflects the electron density. The lower the Wiberg index of double bond $(W)$, the more readily it subdues the attack of radicals. The least polymerization activity of MAA molecules is found in Complex II (Table I). Addition of water to the solvent (formation of Complexes IV and III) increases the activity of MAA molecules; in Complex III it differs only slightly from the activity of noncomplexated MAA molecules.

Regarding the chemical activity of the radical as the concentration of an unpaired electron in definite space, the spin density $(S)$ of the electron reflects this concentration. The higher the spin density, the more active is the radical. According to the data of Table II, the greatest activity of the MAA radical is observed in polymerization in DMSO (Complex II) and the lowest-in water (Complex III). However, it is quite possible that the activity of the acid radical in DMSO may be decreased by some other causes, e.g., a de- crease in spin density of the unpaired electron due to the formation of $\pi$-complex with the solvent, as proposed in this work. ${ }^{24}$

Thus, a significant decrease in the polymerization activity of unsaturated acids in strong electron-donor solvents (e.g., DMSO) mainly depends on two simultaneously occurring effects: the formation of $\mathrm{H}$-complexes between acid molecules and the solvent, and $\pi$ complexes between acid radicals and solvent molecules. Introduction of water into reaction mixture increases the activity of acid molecules in $\mathrm{H}$-complexes and decreases the

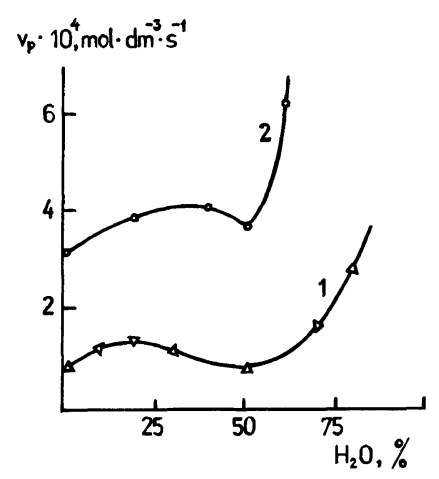

Figure 7. Dependence of copolymerization rate of AN-IA (1) and AN-MAA (2) on the composition of mixed solvent DMSO-water. Monomer feed AN : IA = $90: 10 \mathrm{~mol} \%$, AN : MAA $=80: 20 \mathrm{~mol}^{\circ} \%$.

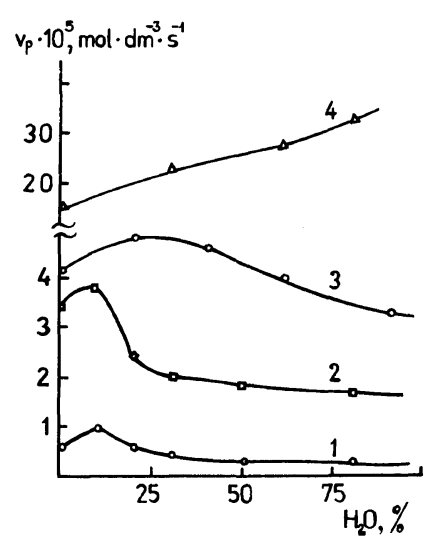

Figure 8. Dependence of copolymerization rate of MAN-IA $(1,2)$ and MAN-MAA $(3,4)$ on the composition of mixed solvent DMSO-water. Monomer feed MAN:IA $=30: 70 \mathrm{~mol} \%$ (1) and $90: 10 \mathrm{~mol} \%$ (2), MAN : MAA $=80: 20 \mathrm{~mol}^{\circ}$ (3) and $20: 80 \mathrm{~mol} \%$ (4). 
Polymerization of Unsaturated Acids in Complex Solutions

Table III. Reactivity ratios of comonomers $\left(r_{1}\right.$ and $\left.r_{2}\right)$ and azeotrope composition of monomer feed $\left(f_{1}\right.$ and $\left.f_{2}\right)$ in copolymerization of MAN with carboxylic acids in DMSO-water solutions

\begin{tabular}{lcccccc}
\hline $\begin{array}{c}\text { Comonomers, } \\
\mathrm{M}_{1}-\mathrm{M}_{2}\end{array}$ & $\begin{array}{c}\text { Solvent, } \\
\text { DMSO }: \mathrm{H}_{2} \mathrm{O} / \%\end{array}$ & $r_{1}$ & $r_{2}$ & $1 / r_{1}$ & $f_{1}$ & $f_{2}$ \\
\hline MAN-AA & $100: 0$ & $2.37 \pm 0.26$ & $0.38 \pm 0.06$ & 0.42 & - & - \\
MAN-AA & $80: 20$ & $1.82 \pm 0.15$ & $0.43 \pm 0.06$ & 0.55 & - & - \\
MAN-AA & $50: 50$ & $1.35 \pm 0.32$ & $0.44 \pm 0.05$ & 0.74 & - & - \\
MAN-MAA & $100: 0$ & $0.72 \pm 0.06$ & $0.50 \pm 0.04$ & 1.39 & 0.64 & 0.36 \\
MAN-MAA & $80: 20$ & $0.57 \pm 0.06$ & $0.66 \pm 0.07$ & 1.75 & 0.44 & 0.56 \\
MAN-MAA & $50: 50$ & $0.53 \pm 0.06$ & $0.91 \pm 0.12$ & 1.89 & 0.16 & 0.84 \\
MAN-IA & $100: 0$ & $1.21 \pm 0.14$ & $0.02 \pm 0.01$ & 0.83 & - & - \\
MAN-IA & $90: 10$ & $0.99 \pm 0.04$ & $0.07 \pm 0.03$ & 1.01 & 0.07 & 0.93 \\
MAN-IA & $80: 20$ & $0.69 \pm 0.04$ & $0.08 \pm 0.05$ & 1.45 & 0.07 & 0.93 \\
\hline
\end{tabular}

probability for the formation of $\pi$-complexes which promote the rate of polymerization.

A more detailed analysis of the changes of the activity of unsaturated carboxylic acids in DMSO- $\mathrm{H}_{2} \mathrm{O}$ solutions is presented in an example of copolymerization of AA, MAA, IA with AN, and MAN. It is known ${ }^{9}$ that from the point of view of complexation through the $\mathrm{H}$-bonds, the nitriles in the above media can be considered as inert components.

Figures 7 and 8 show the changes in the initial rate of copolymerization $\left(V_{\mathrm{p}}\right)$ of MAA or IA with AN and MAN, depending on the mixed solvent. It is evident that IA as a monomer having great steric hindrance is less active in copolymerization (Figures 7, 8, Table III) than MAA or AA. ${ }^{27}$ However in all cases, addition of water to DMSO promotes the rate of copolymerization and increases the contents of acid chains in copolymers (Figure 9). This, of course, depends on more active forms of associated acids, i.e., specifically those interacting with the solvent. It has been shown above that during copolymerization of AA or MAA, complexes IV and III possessing active monomers, are formed. In IA solutions, small amounts of water, apparently, do not impair the established equilibrium between the complexes VI and VII, but are taken into their composition via the $\mathrm{H}$-bonds with carbonyl oxygen. Taking into account quantum-chemical calculations, the formation of acid $\mathrm{H}$-complex with water via $\mathrm{C}=\mathrm{O}$ group increases the activity of monomers.

On enriching monomer mixtures with nitriles, deterioration of the thermodynamic quality of the reaction medium (an increase of water content in the solvent) in copolymerization of MAA or AA results in a decrease of copolymerization rate (Figures 7, 8). A decrease of $V_{\mathrm{p}}$ in the region of the onset of heterophaseness can be explained by the spinning of growing macroradicals leading to the hindrance of interactions with monomer molecules, thus diminishing the propagation rate constant $k_{\mathrm{p}}{ }^{28,29}$ and at the same time $k_{\mathrm{t}}$ undergoes less changes due to the macroradicals being not occluded yet. In heterophase conditions of synthesis (under excess of water in a mixed solvent) $V_{\mathrm{p}}$ increases rapidly in case of copolymerization of AN-MAA (Figure 7). This, apparently, depends on partial ousting of AN into a separate layer, where the conditions of synthesis approximate the analogous ones encountered in producing polymers in mass. Further decrease of $V_{\mathrm{p}}$ in copolymerization of MAN-MAA in heterophase conditions is, to our mind, associated with the peculiarities of synthesis of MAN copolymers mainly in water media and an increase in the chain transfer to monomer and solvation effects. ${ }^{30} \mathrm{~A}$ decrease of $V_{\mathrm{p}}$ in the area of onset of heterophaseness in the copolymerization of IA is expressed more clearly and it involves 
a wider area than in the case of MAA or AA.

As distinct from the above investigated systems (MAN with MAA or AA) in excess of water in mixed solvent, monomer feed enriched on IA slowly undergoes copolymerization, homophaseness of the medium being preserved throughout the procedure. Evidently, complex and specially bulky associates of IA with water fail to promote the monomer reactivity.

The amount of water in the solvent composition determines not only the rate of copolymerization, but also the reactivity ratio of comonomers, and at the same time the composition of copolymers. In the copolymerization of MAN with AA, MAA or IA, an increase in water content in the mixed solvent results in the formation of enriched copolymers on acid chains (Figure 9), and an increase in the reactivity ratio of acids $\left(r_{2}\right)$, and in the value of $1 / r_{1}$, the latter characterizes the activity of acid molecules in relation to MAN radicals (Table III). This affords additional possibilities for synthesis of copolymers when trying to obtain proper compositional characteristics. Particularly, in the copolymerization of MAN and MAA, an increase in the amount of water in the solvent displaces the azeotrope point of monomer mixture in the area where the amount of MAN is less.

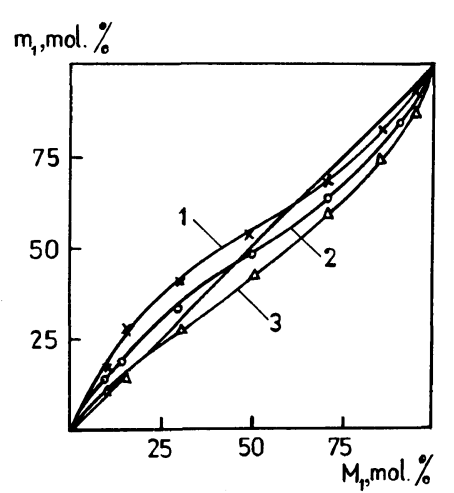

Figure 9. Dependence of copolymer composition on monomer feed during copolymerization of MAN $\left(\mathrm{M}_{1}\right)$ and MAA in DMSO (1), DMSO: $\mathrm{H}_{2} \mathrm{O}=80: 20 \%$ (2) and DMSO: $\mathrm{H}_{2} \mathrm{O}=50: 50 \%$ (3).
Thus, by altering the solvent composition, it is possible to carry out copolymerization in the area of azeotrope, producing homogenous copolymers of a definite composition at high conversions. It is of importance to note that in copolymerization close to the azeotrope point, there is no heterophase which frequently hinders the production of copolymers of homogenous composition (in solvents enriched on water the azeotrope point is shifted to the side rich in acid).

In analyzing the systems of MAN-IA it has been noted that the calculated reactivity ratio of comonomers is insufficient to describe the experimental points. In designing the systems it has been established that one is unable to fairly describe the experimental data by any $r_{1}$ and $r_{2}$ values in a definite interval of meaning. This is possible only in the case when $r_{1}$ and $r_{2}$ undergo changes which depend on the monomer feed. ${ }^{31}$ In fact, the study of the dependence in the coordinates of Fineman and Ross $^{32,33}$ has shown that the dependence has clearly extreme character (Figure 10). In case of copolymerization of monocarboxylic acids (AA, MAA), the dependence of $r_{1}$ and $r_{2}$ on the composition of monomer mixture has not been observed, though there have been such indications before ${ }^{31}$ pointing to the necessity

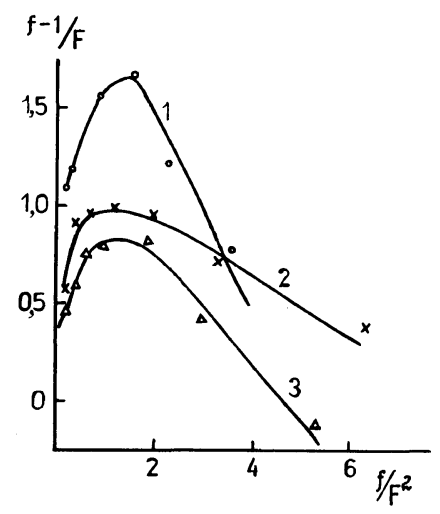

Figure 10. Dependence in the coordinates of Fineman-Ross for the system MAN-IA during copolymerization in DMSO (1), DMSO : $\mathrm{H}_{2} \mathrm{O}=90: 10 \%$ (2) and DMSO: $\mathrm{H}_{2} \mathrm{O}=80: 20 \%$ (3). 
to take into account the "particular" character, i.e., instability of $r_{1}$ and $r_{2}$, in describing the copolymerization systems in which complexation of monomers takes place. Evidently, in the case of IA as a dicarboxylic acid, the competitive complexation mostly depends on its concentration in the solution. Values $r_{1}$ and $r_{2}$ for the monomer systems, including IA, can be regarded only as tentative ones. It cannot be ruled out that in such systems the values $r_{1}$ and $r_{2}$ might differ depending on the conversion of monomers due to a decrease in the amount of monomer acid in the solution.

In this paper an attempt was made to investigate the possible role of complexation of monomers with the medium in copolymerization of unsaturated carboxylic acids with nitriles. It is no exception that the peculiarities of copolymerization of AA, MAA, and IA in DMSO-water solutions depend on some other causes. The main subject of debate is the role of solvation effects of active reaction medium which requires special study.

Acknowledgment. The authors are grateful to Prof. A. Smolyanskii for his assistance in interpreting the results of spectroscopic investigations.

\section{REFERENCES}

1. A. V. Ryabov, J. D. Semchikov, and N. N. Slavnitskaya, Dokl. Akad. Nauk SSSR, 145, 822 (1962).

2. R. Kerber, Makromol. Chem., 96, 30 (1966).

3. A. F. Nikolayev and V. M. Galperin, Vysokomol. Soedin., Ser. A, 9, 2469 (1967).

4. H. Herma, Faserforsch. und Textiltechn., 18, 328 (1967).

5. V. F. Gromov and P. M. Khomikovskii, Usp. Khimii, 48, 1943 (1979).

6. R. Kerber and H. Glamann, Makromol. Chem., 100, 290 (1967).

7. R. Kerber and H. Glamann, Makromol. Chem., 144, 1 (1971).

8. N. A. Kuznetsov and A. L. Smolyanskii, Vysokomol. Soedin., Ser. B, 16, 335 (1974).

9. G. J. Bajoras, G. V. Gusakova, R. J. Makuška, Z. A. Roganova, and A. L. Smolyanskii, Vysokomol. Seodin., Ser. A, 25, 1496 (1983).

10. R. J. Makuška, G. J. Bajoras, J. K. Šulskus, A. B. Bolotin, Z. A. Roganova, and A. L. Smolyanskii,
Vysokomol. Soedin., Ser. A, 27, 567 (1985).

11. J. A. Ustyniuk, Ed., "Quantum-Chemical Methods of Calculation of Molecules," Khimiya, Moscow, 1980, p 256 (in Russian).

12. A. I. Kitaigorodskii, P. M. Zorkii, and V. P. Belskii, "Structure of Organic Substance," Nauka, Moscow, 1980, p 648 (in Russian).

13. L. Pauling and G. Pauling, "Chemistry," Mir, Moscow, 1978, p 688 (transl. in Russian).

14. A. I. Yezriyelew, E. L. Brokhina, and E. S. Roskin, Vysokomol. Soedin., Ser. A, 11, 1670 (1969).

15. S. E. Odinokov, A. V. Yoghansen, and A. K. Dzizenko, J. Priklad. Spectroskopii, 14, 418 (1971).

16. S. V. Gusakova, G. S. Denisov, A. L. Smolyanskii, and V. M. Shraiber, Dokl. Akad. Nauk SSSR, 193, 1065 (1970).

17. G. V. Gusakova, G. S. Denisov, and A. L. Smolyanskii, J. Priklad. Spectroskopii, 14, 860 (1971).

18. G. V. Gusakova and A. L. Smolyanskii, J. Phys. Khimii, 49, 579 (1975).

19. S. Nagai, Bull. Chem. Soc. Jpn., 36, 1459 (1963).

20. S. Nagai and K. Yoshida, Kubunshi Kagaku, 17, 748 (1960).

21. N. S. Golubev, G. S. Denisov, and V. M. Shraiber, in “The Hydrogen Bond," Nauka, Moscow 1981, p 212 (in Russian).

22. G. C. Pimental and A. L. McClellan, "The Hydrogen Bond," Mir, Moscow, 1964, p 158 (transl. in Russian).

23. M. I. Shakhparonov, "Introduction to the Current Theory of Solutions," Vysshaya Shkola, Moscow, 1976, p 296 (in Russian).

24. N. I. Galperina, T. A. Gugunava, V. F. Gromov, P. M. Khomikovskii, and A. D. Abkin, Vysokomol. Soedin., Ser. A, 17, 1455 (1975).

25. G. J. Bajoras, T. N. Ališauskienè, N. N. Slavnitskaya, V. G. Elson, and B. A. Yegorov, Vysokomol. Soedin., Ser. B, 23, 86 (1981).

26. R. Zagradnik and G. Polak, "Principles of Quantum Chemistry," Mir, Moscow, 1979, p 504 (in Russian).

27. D. V. Cicenaité, O. D. Jakubauskaite, and G. J. Bajoras, "Physico-Chemical Principles of Synthesis and Production of Polymers," Interscool Ed., Ser. 2, Gorky University, 1977, p 14 (in Russian).

28. G. Vidotto, A. Crosato-Arnaldi, and G. Talamini, Makromol. Chem., 122, 91 (1969).

29. M. Ryska, M. Kolinsky, and D. Lim, J. Polym. Sci., $C, 16,621$ (1967).

30. R. J. Makuška, Dissertation for Candiate Chemical Science degree, Vilnius University, 1983.

31. V. A. Myagchenkov and S. Ya. Frenkel, Usp. Khimii, 47, 1261 (1978).

32. M. Fineman and S. D. Ross, J. Polym. Sci., 5, 259 (1950).

33. V. A. Myagchenkov and S. Ya. Frenkel, Dokl. Akad. Nauk SSSR, 186, 631 (1969). 